\title{
ANALYTIC SOLUTION OF RICCATI EQUATIONS OCCURRING IN OPEN-LOOP NASH MULTIPLAYER DIFFERENTIAL GAMES
}

\author{
L. JÓDAR and E. NAVARRO \\ Departamento de Matemática Aplicada \\ Universidad Politécnica de Valencia \\ E.T.S.I.I., P.O. Box 22.012 \\ Valencia, Spain
}

(Received January 2, 1991)

\begin{abstract}
In this paper we present explicit analytic solutions of coupled Riccati matrix differential systems appearing in open-loop Nash games. Two different cases are considered. Firstly, by means of appropriate algebraic transformations the problem is decoupled so that an explicit solution of the problem is available. The second is based on the existence of a solution of a rectangular Riccati type algebraic matrix equation associated with the problem.
\end{abstract}

KEY WORDS AND PHRASES. Riccati equations, differential games, optimization problem. 1991 AMS SUBJECT CLASSIFICATION CODE. 34A34

1. INTRODUCTION. When noncooperative problems are tackled, a game theoretic approach is necessary: each control agent (decision maker or player) tries to optimize his own cost function which conflicts more or less with others. An equilibrium solution must be sought, and the Nash strategy is a natural choice. In this case, a player cannot improve his payoff by deviating unilaterally from his Nash strategy. Due to this noncooperation, the optimization problem of various players are strongly coupled and necessary conditions for open-loop Nash strategy lead to complex two-point boundary value problems.

Consider a $N$-players linear quadratic differential game defined by

$$
x^{\prime}=A x+\sum_{i=1}^{N} B_{i} u_{i} ; \quad x(0)=x_{0}
$$

with the cost functionals associated with the players

$$
J_{i}=\frac{1}{2}\left\{x_{f}^{T} K_{i f} x_{f}+\int_{0}^{t_{f}}\left(x^{T} Q_{i} x+\sum_{j=1}^{N} u_{j}^{T} R_{i j} u_{j}\right) d t\right\} ; \quad x\left(t_{f}\right)=x_{f}
$$

where all matrices are $n \times n$ symmetric with $R_{i i}$, for $1 \leq i \leq N$, positive definite. It is well known that the open-loop Nash control must satisfy [12]:

$$
u_{i}=-R_{i i}^{-1} B_{i}^{T} \Psi_{i} ; \quad \Psi_{i}^{\prime}=-Q_{i} x-A^{T} \Psi_{i}, \quad \Psi_{i}\left(t_{f}\right)=K_{i f} x_{f}, \quad 1 \leq i \leq N
$$

where $\Psi_{i}$ is the costate vector associated with player " $i$ ". When the transformation $\Psi_{i}=K_{i} x$, is introduced, for $1 \leq i \leq N$, the open-loop Nash strategy $\left(u_{i}^{*}\right)_{i=1}^{N}$ is given by

$$
u_{i}^{*}=-R_{i i}^{-1} B_{i}^{T} K_{i}(t) \Phi(t, 0) x_{o}, \quad 1 \leq i \leq N
$$


where

with

$$
K_{i}^{\prime}=-A^{T} K_{i}-K_{i} A-Q_{i}+K_{i} \sum_{j=1}^{N} S_{j} K_{j} ; \quad K_{i}\left(t_{f}\right)=K_{i f}, \quad 1 \leq i \leq N
$$

$$
S_{i}=B_{i} R_{i i}^{-1} B_{i}^{T}, \quad 1 \leq i \leq N
$$

and $\Phi(t, 0)$ is the system's transition matrix satisfying

$$
\Phi^{\prime}(t, 0)=\left(A-\sum_{j=1}^{N} S_{j} K_{j}\right) \Phi(t, 0) ; \quad \Phi(t, t)=I
$$

Note that the matrices $R_{i j}$ for $i \neq j$ do not appear in the necessary conditions due to the fact that under open-loop strategy assumptions, each player optimizes his criterion knowing that $\partial u_{i} / \partial x=0$, for $1 \leq i \leq N$. For the open-loop Nash strategy and under the existence of a solution of the coupled Riccati system, the optimization problem has only one solution [2].

The solution of system (1.5) is generally difficult to obtain due to the permanent coupling between the players' strategies. In [4] a series solution of system (1.5) is proposed but the coefficient are obtained solving several linear matrix equations. In [14] a numerical algorithm for the integration of (1.5) is given. A singular perturbation method for solving (1.5) is proposed in [10]. For the case $N=2$, an iterative algorithm for solving (1.5) is given in [5]. For the case $N=2$ and $Q_{2}=\alpha Q_{1}$, where $\alpha$ is a scalar, an analytic solution of system (1.5) was pointed out in [1]. In this paper we obtain an explicit solution of system (1.5) for a case more general than the one proposed in [1]. Also, a different type condition expressed in terms of the existence of certain coupled algebraic Riccati matrix system is proposed.

2. ANALYTIC SOLUTION OF COUPLED RICCATI SYSTEM BASED ON ALGEBRAIC TRANSFORMATIONS.

For convenience, the necessary conditions to be satisfied (1.1), (1.3), are rewritten in a matrix form as

$$
\begin{gathered}
{\left[\begin{array}{c}
x^{\prime} \\
\Psi_{1}^{\prime} \\
\Psi_{2}^{\prime} \\
\vdots \\
\Psi_{N}^{\prime}
\end{array}\right]=\left[\begin{array}{ccccc}
A & -S_{1} & -S_{2} & \cdots & -S_{N} \\
-Q_{1} & -A^{T} & 0 & \cdots & 0 \\
-Q_{2} & 0 & -A^{T} & \cdots & 0 \\
\vdots & & & & \vdots \\
-Q_{N} & 0 & 0 & \cdots & -A^{T}
\end{array}\right]\left[\begin{array}{c}
x \\
\Psi_{1} \\
\Psi_{2} \\
\vdots \\
\Psi_{N}
\end{array}\right]=M\left[\begin{array}{c}
x \\
\Psi_{1} \\
\Psi_{2} \\
\vdots \\
\Psi_{N}
\end{array}\right]} \\
x(0)=x_{o}, \quad \Psi_{i}\left(t_{f}\right)=K_{i f} x_{f}, \quad 1 \leq i \leq N
\end{gathered}
$$

Now, let us introduce the change of basis

$$
\left[\begin{array}{c}
x \\
\Psi_{1} \\
\Psi_{2} \\
\vdots \\
\Psi_{N}
\end{array}\right]=T\left[\begin{array}{c}
x \\
\Psi_{1} \\
w_{2} \\
\vdots \\
w_{N}
\end{array}\right] ; \quad T=\left[\begin{array}{ccccc}
I & 0 & 0 & \ldots & 0 \\
0 & I & 0 & \ldots & 0 \\
0 & L_{2} & I & \ldots & 0 \\
\vdots & \vdots & & & \vdots \\
0 & L_{N} & 0 & \ldots & I
\end{array}\right]
$$

for appropriate matrices $L_{2}, L_{3}, \cdots, L_{N}$ in $\mathbb{R}^{n \times n}$ to be determined. Thus problem (2.1), (2.2) is 
equivalent to the following one

$$
\begin{gathered}
{\left[\begin{array}{c}
x^{\prime} \\
\Psi_{1}^{\prime} \\
w_{2}^{\prime} \\
\vdots \\
w_{N}
\end{array}\right]=\left[\begin{array}{ccccc}
A & -S_{1}-S_{2} L_{2}-\cdots-S_{N} L_{N} & -S_{2} & \cdots & -S_{N} \\
-Q_{1} & -A^{T} & 0 & \cdots & 0 \\
L_{2} Q_{1}-Q_{2} & L_{2} A^{T}-A^{T} L_{2} & -A^{T} & \cdots & 0 \\
\vdots & \vdots & \vdots & \vdots \\
L_{N} Q_{1}-Q_{N} & L_{N} A^{T}-A^{T} L_{N} & 0 & \cdots-A^{T}
\end{array}\right]\left[\begin{array}{c}
x \\
\Psi_{1} \\
w_{2} \\
\vdots \\
w_{N}
\end{array}\right]} \\
x(0)=x_{o}, \quad \Psi_{1}\left(t_{f}\right)=K_{1 f} x_{f}, \quad w_{j}\left(t_{f}\right)=\left(K_{j f}-L_{j} K_{1 f}\right) x_{f}, \quad 2 \leq j \leq N
\end{gathered}
$$

The purpose of this transformation is to find under what conditions the player's optimization problem can be decoupled. In fact, note that if $L_{2}, L_{3}, \ldots, L_{N}$ satisfy the system

$$
L_{j} Q_{1}=Q_{j}, \quad L_{j} A^{T}=A^{T} L_{j}, \quad 2 \leq j \leq N
$$

the matrix $T^{-1} M T$ is reduced to a block triangular form and the costate vectors $\Psi_{1}, w_{2}, \cdots, w_{N}$, are coupled only via the terminal conditions (2.2).

Note that for the case $N=2$ and the matrices $Q_{1}$ and $Q_{2}$ are proportional, i.e., $Q_{2}=\alpha Q_{1}$, for some scalar $\alpha$, taking $L=\alpha I$, one gets solutions of system (2.6) for $N=2$. Thus the case studied in [1] is a particular case of (2.6).

In order to characterize the existence of solutions for the algebraic system (2.6), we recall the concept of tensor product of matrices. If $A, B$ are matrices in $\mathbf{R}^{m \times n}$ and $\mathbb{R}^{k \times s}$, respectively, then the tensor product of $A$ and $B$, denoted $A \otimes B$, is defined as the partitioned matrix

$$
A \otimes B=\left[\begin{array}{cccc}
a_{11} B & a_{12} B & \cdots & a_{1 n} B \\
\vdots & & & \vdots \\
a_{m 1} B & a_{m 2} B & \cdots & a_{m n} B
\end{array}\right]
$$

If $A \in \boldsymbol{R}^{m \times n}$, we denote

$$
A_{\cdot j}=\left[\begin{array}{c}
a_{1 j} \\
\vdots \\
a_{m j}
\end{array}\right], 1 \leq j \leq n ; \quad \operatorname{vec} M=\left[\begin{array}{c}
M_{\cdot 1} \\
\vdots \\
M \cdot n
\end{array}\right]
$$

If $M, N$ and $P$ are matrices of suitable dimensions, then using the column lemma [7, p. 410], we get

$$
\operatorname{vec}(M N P)=\left(P^{T} \otimes M\right) \operatorname{vec} N
$$

Taking into account (2.7), if we apply tensor products in each equation of system (2.6), it may be written in the equivalent form

$$
C \operatorname{vec} L_{j}=\operatorname{vec}\left[0, Q_{j}\right]
$$

where $2 \leq j \leq N$ and

$$
C=\left[\begin{array}{c}
I \otimes A^{T}-A \otimes I \\
Q_{1} \otimes I
\end{array}\right]
$$

If we denote by $C^{+}$the Moore-Penrose pseudoinverse of $C$, then from Theorem 2.3.2 of [11, p.24], 
the system (2.9) is compatible if and only if

$$
C C^{+} \operatorname{vec}\left[0, Q_{j}\right]=\operatorname{vec}\left[0, Q_{j}\right]
$$

Furthermore, under condition (2.10), the general solution of (2.9) for $L_{j}$ is given by

$$
\operatorname{vec} L_{j}=C^{+} \operatorname{vec}\left[0, Q_{2}\right]+\left(I-C^{+} C\right) z
$$

where $I$ denotes the identity matrix in $R^{n^{2}} \times^{n^{2}}$ and $z$ is an arbitrary vector in $R^{n^{2}}$. We recall that $C^{+}$may be computed by using MATLAB [8].

Let us assume the existence of solutions $L_{j}$ of $(2.8)$ for $2 \leq j \leq N$, then from (2.4), (2.5) it follows that

$$
\left[\begin{array}{c}
x^{\prime} \\
\Psi_{1}^{\prime} \\
w_{2}^{\prime} \\
\vdots \\
w_{N}^{\prime}
\end{array}\right]=\left[\begin{array}{ccccc} 
& 1 & -S_{2} & \cdots & -S_{N} \\
& 1 & 0 & \cdots & 0 \\
\cdots & 1 & -A^{T} & \cdots & 0 \\
0 & & \vdots & \ddots & \vdots \\
& 1 & 0 & \cdots & -A^{T}
\end{array}\right]\left[\begin{array}{c}
x \\
\Psi_{1}^{\prime} \\
w_{2}^{\prime} \\
\vdots \\
w_{N}^{\prime}
\end{array}\right]
$$

$$
x(0)=x_{0} \quad \Psi_{1}\left(t_{f}\right)=K_{1 f} x_{f} \quad w_{j}\left(t_{f}\right)=\left(K_{j f}-L_{j} K_{1 f}\right) x_{f} \quad 2 \leq j \leq N
$$

where

$$
V=\left[\begin{array}{cc}
A & -S_{1}-S_{2} L_{2}-\cdots-S_{N} L_{N} \\
-Q_{1} & -A^{T}
\end{array}\right]
$$

Let us consider the change $t=t(s)=t_{f}-s, 0 \leq s \leq t_{f}$, and let

$$
\begin{aligned}
\widehat{x}(s) & =x\left(t_{f}-s\right)=x(t) \\
\widehat{\Psi}_{1}(s) & =\Psi_{1}\left(t_{f}-s\right)=\Psi_{1}(t) \\
\widehat{w}_{j}(s) & =w_{j}\left(t_{f}-s\right)=w_{j}(t), \quad 2 \leq j \leq N
\end{aligned}
$$

Hence problem (2.11) may be written in the form

$$
(d / d s)\left[\begin{array}{c}
\widehat{x} \\
\hat{\Psi}_{1} \\
\widehat{w}_{2} \\
\vdots \\
\widehat{w}_{N}
\end{array}\right]=\left[\begin{array}{ccccc} 
& 1 & -S_{2} & \cdots & -S_{N} \\
-V & & & & \\
& 1 & 0 & \cdots & 0 \\
\cdots & 1 & -A^{T} & \cdots & 0 \\
& 1 & 0 & \cdots & -A^{T}
\end{array}\right]\left[\begin{array}{c}
\widehat{x} \\
\widehat{\Psi}_{1} \\
\hat{w}_{2} \\
\vdots \\
\widehat{w}_{N}
\end{array}\right]
$$




$$
\widehat{x}\left(t_{f}\right)=x_{o}, \quad \widehat{\Psi}_{1}(0)=K_{1 f} \widehat{x}(0), \quad \widehat{w}_{j}(0)=\left(K_{j f}-L_{j} K_{1 f}\right) \widehat{x}(0), \quad 2 \leq j \leq N
$$

Solving (2.15) we obtain

$$
\begin{gathered}
\widehat{w}_{j}(s)=\exp \left(s A^{T}\right) \widehat{w}_{j}(0), \quad 2 \leq j \leq N \\
{\left[\begin{array}{c}
\widehat{x}(s) \\
\widehat{\Psi}_{1}(s)
\end{array}\right]=\exp (-s V)\left\{\left[\begin{array}{c}
\widehat{x}(0) \\
\hat{\Psi}_{1}(0)
\end{array}\right]+\sum_{j=2}^{N} \int_{0}^{s} \exp (u V)\left[\begin{array}{c}
S_{j} \\
0
\end{array}\right] \exp \left(u A^{T}\right) \widehat{w}_{j}(0) d u\right\}}
\end{gathered}
$$

From (2.14) and (2.16) we have

$$
\left[\begin{array}{c}
\widehat{x}(s) \\
\widehat{\Psi}_{1}(s)
\end{array}\right]=G(s) \widehat{x}(0)
$$

where

Thus we have

$$
G(s)=\exp (-s V)\left\{\left[\begin{array}{c}
I \\
K_{1 f}
\end{array}\right]+\sum_{j=2}^{N} \int_{0}^{s} \exp (u V)\left[\begin{array}{c}
S_{j} \\
0
\end{array}\right] \exp \left(u A^{T}\right) d u\left(K_{j f}-L_{j} K_{1 f}\right)\right\}
$$

$$
\widehat{x}(s)=[I, 0] G(s) \widehat{x}(0) ; \quad \widehat{\Psi}_{1}(s)=[0, I] G(s) \widehat{x}(0)
$$

Note that $[I, 0] G(0)=I$, and from the continuity of $G$, there exists an interval $0 \leq s \leq \delta$, such that

$$
[I, 0] G(s) \quad \text { is invertible for all } s \in[0, \delta]
$$

From (2.19) and (2.20) we obtain

$$
\begin{gathered}
\widehat{x}(0)=\{[I, 0] G(s)\}^{-1} \widehat{x}(s) \\
\widehat{\Psi}_{j}(s)=[0, I] G(s)\{[I, 0] G(s)\}^{-1} \widehat{x}(s), \quad 0 \leq s \leq \delta
\end{gathered}
$$

Now, from (2.3) and (2.13), it follows that

$$
\hat{\Psi}_{j}(s)=L_{j} \widehat{\Psi}_{1}(s)+\widehat{w}_{j}(s), \quad 2 \leq j \leq N
$$

and from (2.15) and (2.19)

$$
\widehat{\Psi}_{j}(s)=\left\{\exp \left(s A^{T}\right)\left(K_{j f}-L_{j} K_{l f}\right)+L_{j}[0, I] G(s)\right\}\{[I, 0] G(s)\}^{-1} \widehat{x}(s)
$$

for $0 \leq s \leq \delta$.

From (2.13) and the relations $\Psi_{j}(t)=K_{j}(t) x(t), 1 \leq j \leq N$, it follows that

$$
\begin{gathered}
K_{1}(t)=[0, I] G\left(t_{f}-t\right)\left\{[I, 0] G\left(t_{f}-t\right)\right\}^{-1} \\
K_{j}(t)=\left\{\exp \left(A^{T}\left(t_{f}-t\right)\right)\left[K_{j f}-L_{j} K_{1 f}\right]+L_{j}[0, I] G\left(t_{f}-t\right)\right\}\left\{[I, 0] G\left(t_{f}-t\right)\right\}^{-1}, \\
t_{f}-\delta \leq t \leq t_{f} ; \quad 2 \leq j \leq N
\end{gathered}
$$

where $G$ is defined by (2.18). Thus the following result has been proved:

THEOREM 1. Let us assume that matrices $A$ and $Q_{i}$ for $1 \leq i \leq N$, satisfy the condition (2.9) where $C$ is defined by (2.8), and let $L_{j}$ be the solution of (2.8) for $2 \leq j \leq N$. Then there exists a positive number $\delta$ such that on the interval $\left[t_{f}-\delta, t_{f}\right]$ the unique solution of the coupled Riccati system (1.5) is given by (2.23), (2.24). 
REMARK 1. Note that the case $N=2, Q_{2}=\alpha Q_{1}$, where $\alpha$ is a scalar, is a particular case of the previous theorem taking $L_{2}=\alpha I$. It is important to note that from (2.23) and (2.24), we have the following relation between $K_{1}(t)$ and $K_{2}(t)$ :

$$
K_{2}(t)=L_{2} K_{1}(t)+\exp \left(A^{T}\left(t_{f}-t\right)\right)\left[K_{2 f}-L_{2} K_{1 f}\right]\left\{[I, 0] G\left(t_{f}-t\right)\right\}^{-1}
$$

and since the function $\left\{[I, 0] G\left(t_{f}-t\right)\right\}^{-1}$ is involved in the computation of $K_{1}(t)$, the computational cost is reduced because $K_{2}(t)$ is expressed in terms of $K_{1}(t)$. Finally we recall that efficient methods for computing matrix exponentials and integrals involving them and that appear in the expression of $G(s)$, may be found in [13]. These procedures are extremely easy to implement and yield an estimation of the approximation error.

3. ANALYTIC SOLUTION OF COUPLED RICCATI DIFFERENTIAL SYSTEM BASED ON THE EXISTENCE OF A SOLUTION OF A COUPLED ALGEBRAIC RICCATI SYSTEM.

Riccati type matrix equations with rectangular coefficients arise for instance in the problem of finding a state estimate feedback controller [3] and in the transformation of ill-conditioned linear systems to a block diagonal form [6,9]. An efficient method to find solutions of such equations may be found in [15]. The aim of this section is to propose another way to find an analytic solution of the coupled Riccati differential system (1.5). Note that system (1.5) may be written in the following compact form

$$
K^{\prime}(t)=-Q-K(t) A-B K(t)+K(t) S K(t) ; \quad K\left(t_{f}\right)=K_{f}
$$

where

$$
K=\left[\begin{array}{c}
K_{1} \\
\vdots \\
K_{N}
\end{array}\right] ; \quad Q=\left[\begin{array}{c}
Q_{1} \\
\vdots \\
Q_{N}
\end{array}\right] ; \quad B=\operatorname{Diag}\left(A^{T}, A^{T}, \ldots, A^{T}\right), \quad K_{f}=\left[\begin{array}{c}
K_{1 f} \\
\vdots \\
K_{N f}
\end{array}\right]
$$

and

$$
S=\left[S_{1}, S_{2}, \ldots, S_{N}\right]
$$

Let us assume that the rectangular algebraic Riccati equation

$$
-Q-X A-B X+X S X=0
$$

admits a solution $X_{o} \in \mathrm{C}^{N n \times n}$ and let us consider the change

$$
U(t)=K(t) \equiv X_{o}
$$

Then problem (3.1) is equivalent to the following one

$$
U^{\prime}(t)=B_{o} U(t)-U(t) A_{o}+U(t) S U(t) ; \quad U\left(t_{f}\right)=U_{f}
$$

where

$$
B_{o}=X_{o} S-B ; \quad A_{o}=A-S X_{o} ; \quad U_{f}=K_{f}-X_{o}
$$

Now, let us consider the extended linear system

$$
(d / d t)\left[\begin{array}{c}
V(t) \\
Z(t)
\end{array}\right]=\left[\begin{array}{cc}
A_{o} & -S \\
0 & B_{o}
\end{array}\right]\left[\begin{array}{l}
V(t) \\
Z(t)
\end{array}\right] ; \quad\left[\begin{array}{l}
V\left(t_{f}\right) \\
Z\left(t_{f}\right)
\end{array}\right]=\left[\begin{array}{c}
I \\
U_{f}
\end{array}\right]
$$


where $V(t) \in \mathrm{C}^{n \times n}$ and $Z(t) \in \mathrm{C}^{N n \times n}$. If we define the matrix function

$$
S(t, s)=\left[\begin{array}{ccc}
\exp \left((t-s) A_{o}\right) & -\int_{t_{f}}^{t} \exp \left((t-v) A_{o}\right) \operatorname{Sexp}\left((v-x) B_{o}\right) d v \\
0 & \exp \left((t-s) B_{o}\right)
\end{array}\right]
$$

then an easy computation yields

$$
(\partial / \partial t) S(t, s)=\left[\begin{array}{cc}
A_{o} & -S \\
0 & B_{o}
\end{array}\right] S(t, s)
$$

and thus $S(t, s)$ is a fundamental matrix of (3.8) and the unique local solution of (3.8) in a neighborhood of $t_{f}$ is given by

$$
\left[\begin{array}{l}
V(t) \\
Z(t)
\end{array}\right]=S\left(t, t_{f}\right)\left[\begin{array}{c}
I \\
U_{f}
\end{array}\right] ; \quad S\left(t_{f}, t_{f}\right)=I_{(N+1) n}
$$

Note that $V\left(t_{f}\right)=I_{n}$ and thus in a neighbourhood $J$ containing $t_{f}, V(t)$ is invertible. Now, let us define the $C^{N n \times n}$-values matrix function $U(t)=Z(t)(V(t))^{-1}$ for $t \varepsilon J$. Note that from (3.8) it follows that

$$
V^{\prime}(t)=A_{o} V(t)-S Z(t) \text { and } \quad Z^{\prime}(t)=B_{o} Z(t)
$$

Computing it follows that

$$
U^{\prime}(t)=Z^{\prime}(t)(V(t))^{-1}-Z(t)(V(t))^{-1} V^{\prime}(t)(V(t))^{-1}=B_{0} U(t)-U(t) A_{0}+U(t) S U(t)
$$

for all $t \in J$. Hence $K(t)=U(t)+X_{o}$ is the solution of (3.1), defined on the interval $J$. From (3.10) it follows that

$$
\begin{gathered}
V(t)=\exp \left(\left(t-t_{f}\right) A_{o}\right)-\int_{t_{f}}^{t} \exp \left((t-v) A_{o}\right) \operatorname{sexp}\left(\left(v-t_{f}\right) B_{o}\right) d v U_{f} \\
=\exp \left(\left(t-t_{f}\right) A_{o}\right)\left\{I-\int_{t_{f}}^{t} \exp \left(\left(t_{f}-v\right) A_{o}\right) \operatorname{Sexp}\left(v B_{o}\right) d v \exp \left(-t_{f} B_{o}\right) U_{f}\right\} \\
Z(t)=\exp \left(\left(t-t_{f}\right) B_{o}\right) U_{f} \\
K(t)=X_{o}+\exp \left(\left(t-t_{f}\right) B_{o}\right) U_{f}\left\{I-\int_{t_{f}}^{t} \exp \left(\left(t_{f}-v\right) A_{o}\right) \operatorname{sexp}\left(v B_{o}\right) d v \exp \left(-t_{f} B_{o}\right) U_{f}\right\} \begin{array}{c}
\left.\exp \left(\left(t_{f}-t\right) A_{o}\right)\right) \\
(3.11)
\end{array}
\end{gathered}
$$

Thus if the algebraic Riccati equation (3.4) has a solution $X_{o}$, chen the solution $K(t)$ of (3.1) is defined by (3.11) where $U_{f}, A_{o}$ and $B_{o}$ are given by (3.7). The solution is defined in the neighbourhood of $t_{f}$ where $V(t)$ is invertible.

ACKNOWLEDGEMENT. This paper has been partially supported by the D.G.I.C.Y.T. grant PS87-0064 and the NATO grant 900040.

\section{REFERENCES}

1. ABOU-KANDIL, H. and BERTRAND, P., Analytic solution for a class of linear quadratic open-loop Nash games, Internat. I. Control 43 (1986), 997-1002. 
2. BASAR, T., On the uniqueness of the Nash solution in linear quadratic differential games, Internat. J. of Game Theory, vol. 5 (1973), 65-90.

3. ANDERSON, B.D.O., The testing of optimality linear systems, Internat. J. Control 4 No. 1 (1966), 29-40.

4. CRUZ, J.B., JR. and CHEN, C.I., Series Nash solution of two-person nonzero sum linear quadratic games, J. Opt. Theory and Appl. Z(1971), 240-257.

5. JÓDAR, L. and ABOU-KANDIL, H., A resolution method for Riccati differential systems coupled in their quadratic terms, SIAM J. Math. Anal. 19 (1988), 1225-1230.

6. KOKOTOVIC, P.V., A Riccati equation for block-diagonalization of ill-contditioned systems, IEEE Trans. Autom. Control AC-21(1975), 812-814.

7. LANCASTER, P. and TISMENETSKY, M., The theory of matrices, Second ed. Academic Press, New York, 1985.

8. MOLER, C.B., MATLAB User's guide, Technical Report CS81-1(1980) Department of Computer Science, Univ. of New Mexico, Alburquerque, New Mexico, 87131.

9. NARASIMHAMURTHI, N. and WU, F.F., On the Riccati equation arising from the study of singularly perturbed systems, Proc. of the 1977 Joint Autom. Control Conf. 22-24 June, San Franscisco, Vol. V, 1244-1247.

10. OZGUNER, U. and PERKINS, W.R., A series solution to the Nash strategy for large scale interconnected systems, Automatica, Vol. 13(1977), 313-315.

11. RAO, C.R. and MITRA, S.K., Generalized Inverse of Matrices and its Applications, JohnWiley, New York, 1971.

12. STARR, A.W. and HO, Y.C., Non-zero sum differential games, J. Optimization Theory and Appl.. $3(1969), 179-197$.

13. VAN LOAN, C.F., Computing integrals involving the matrix exponential, IEEE Trans. Aut. Control. AC-23 (1978), 395-404.

14. SIMAAN, M. and CRUZ, J.B., JR., On the solution of open-loop Nash Riccati equations in linear quadratic differential games, Internat, J. Control, Vol. 18 (1973), 57-63.

15. MEYER, H.B., The matrix equation $A Z+B-Z C Z-Z D=0$, SIAM J. Appl. Math. \& Vol. 30. №. 1 (1976), 136-142. 


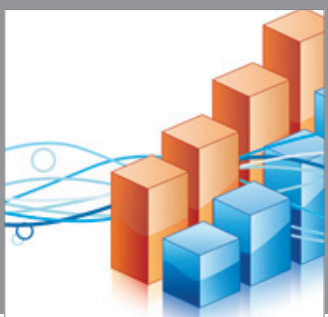

Advances in

Operations Research

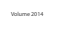

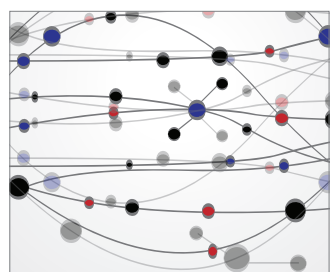

\section{The Scientific} World Journal
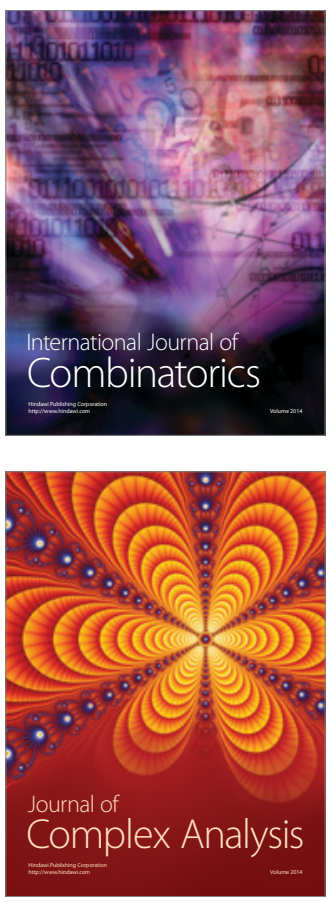

International Journal of

Mathematics and

Mathematical

Sciences
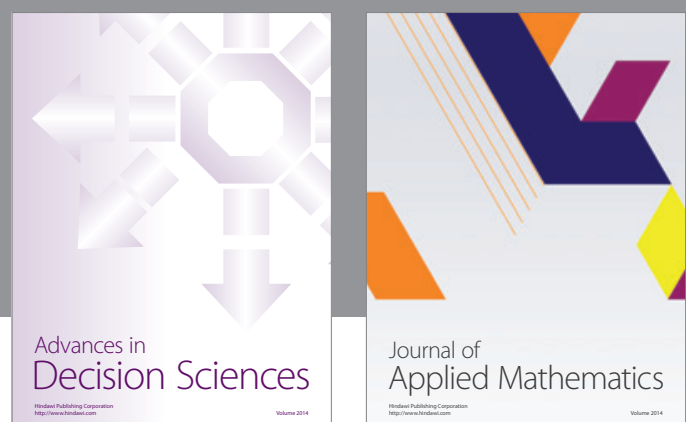

Journal of

Applied Mathematics
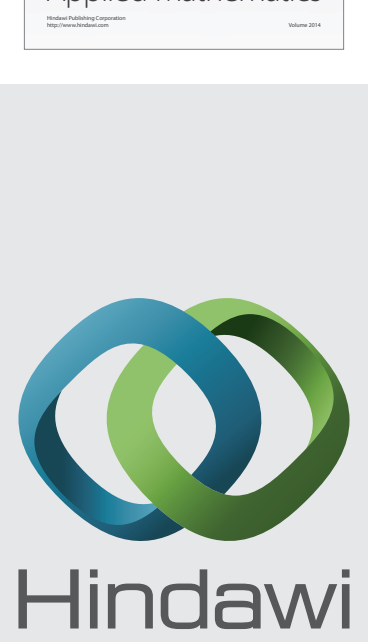

Submit your manuscripts at http://www.hindawi.com
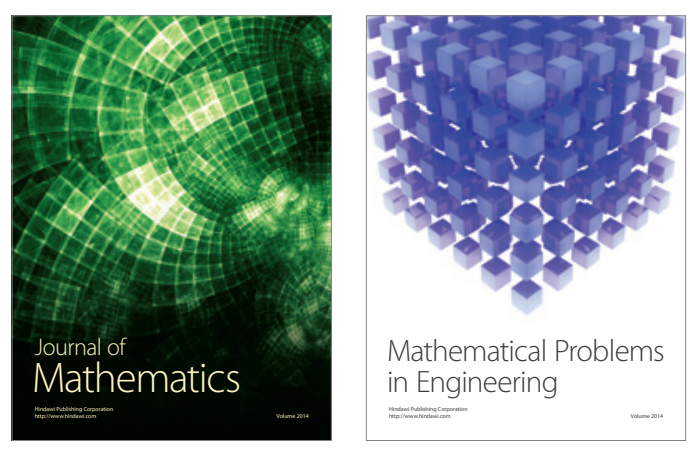

Mathematical Problems in Engineering
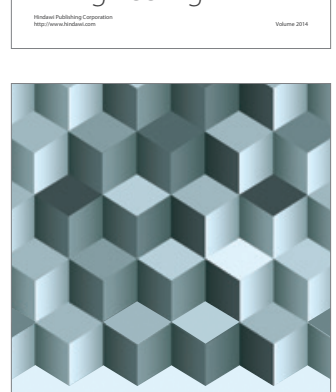

Journal of

Function Spaces
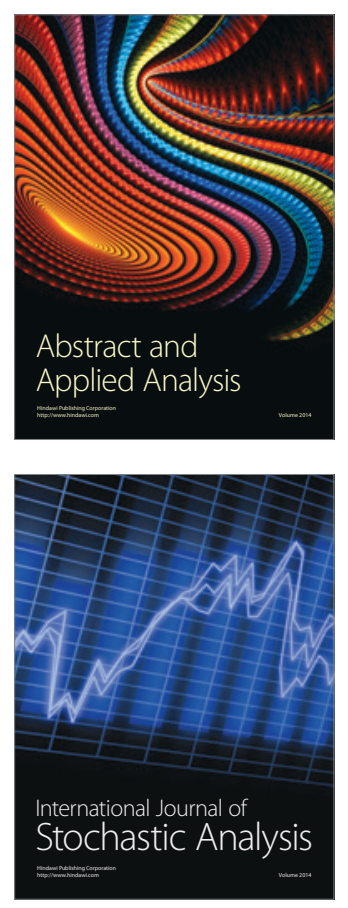

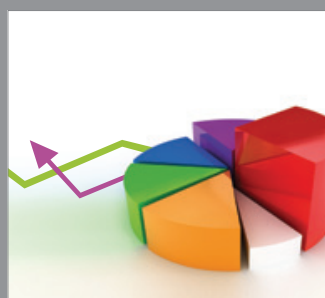

ournal of

Probability and Statistics

Promensencen
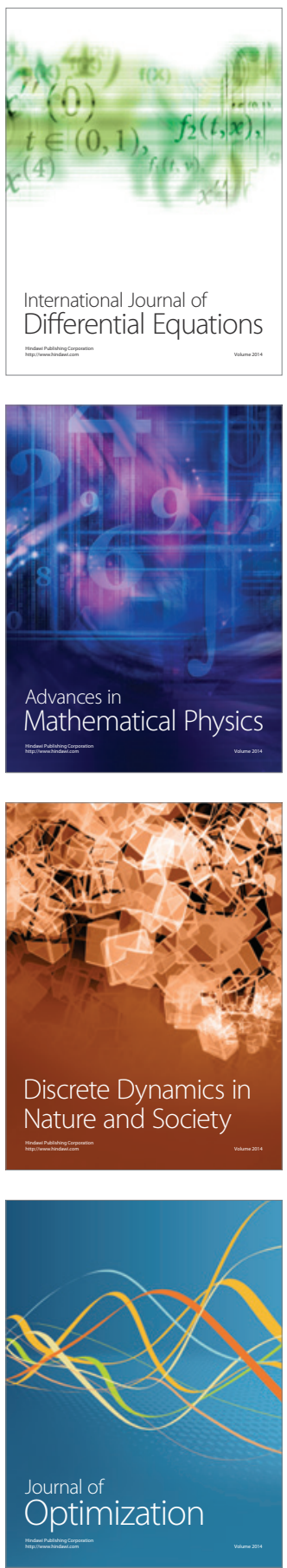